logical mechanism required for a psychiatric disorder? People were depressed long before we knew anything about low serotonin and high cortisol. What's more, the presence of acute stress disorder strongly predicts the development of PTSD. We know a lot about the psychobiological mechanism of PTSD, ranging from diurnal cortisol abnormalities to smaller hippocampal volume.

McNally mistakenly assumes that a diagnosis of obsessive-compulsive disorder rules out the possibility that the patients' intrusive images are true post-traumatic reactions. In fact, one-third of patients with obsessive-compulsive disorder have a traumatic origin to their symptoms. I have seen patients whose obsessive-compulsive symptoms began after sexual abuse.

McNally also states, startlingly, that "the low base rate of HIV infection in the general population means that a positive HIV test will almost always be wrong". Huh? Most doctors (and many patients) would be scratching their heads by now. Sure, even a low false-positive rate can produce many errors in a large uninfected population. But that is not how the test is used. The test will not "almost always be wrong," because it is not used as a population screen, but among people with elevated risk.

McNally bemoans the (remarkably) unanimous Congressional condemnation of the Bruce Rind meta-analysis, which claims no evidence of ill effects of childhood sexual abuse, as "perhaps the most egregious example of (perceived) advocacy trumping science". But he does not mention that Psychological Bulletin published two full-length methodological criticisms of the Rind paper. The problem with that meta-analysis is not just its conclusions, but its assumptions and methods: deficient science, not politics.

Remembering Trauma is a stimulating book. It contains a clear and useful review of experimental and clinical literature on trauma and memory. It could have been even more memorable, however, if the retrieval were as balanced as the encoding.

\section{On the trail of mad cows}

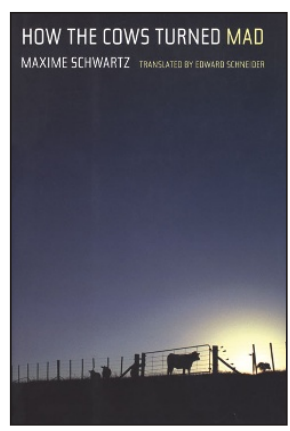

\section{How the Cows Turned Mad}

by Maxime Schwartz

University of California Press, 2003

238 pp. hardcover, $\$ 24.95$

ISBN 0520235312

\section{Reviewed by R Anthony Williamson}

Any good drama has a number of key ingredients: strong characters, intrigue, unexpected plot twists and high stakes. The prion story has these aplenty and, as recounted by Maxime Schwartz, makes a fascinating and engrossing read. This is a thoroughly researched book that is written in an authoritative-but always accessible-style, as one might expect from a former head of the Institut Pasteur. The author instructively and expertly guides us through the complexities of scientific detective work and discovery, successfully interweaving the occasionally disparate threads of a plot that has played out for well over 200 years. It is an excellent introduction to the history of prion diseases, but it may not recite all of the key experiments-or explain the current controversies facing the prion protein-only hypothesis-in sufficient detail to satiate the prion devotee.

We begin our journey at a time in the early nineteenth century when scientific practitioners of the day attributed what we now know to be scrapie prion disease in sheep to the sexual frustration of overly amorous rams. As the pages turn, however, we observe how successive generations of scientific investigators slowly assembled the pieces of a new and deadly puzzle. Educationally, each advance in understanding is placed within the context of the scien-

R. Anthony Williamson is an assistant professor in immunology at The Scripps Research Institute, 10550 North Torrey Pines Road, La Jolla, California 92037, USA.

e-mail: anthony@scripps.edu tific knowledge and techniques of the day. Occasionally the trail peters out, only to be picked up by another investigator in another country, decades later. At several junctions, the author reminds us how the echoes of history can manifest in our modern lives. For example, the spread of anthrax infection in the France of the 1880s was perpetuated by fertilizers prepared from the waste of infected animals. These events are remarkably reminiscent of the role of animal feed in the dissemination of bovine spongiform encephalopathy (BSE) prions in cattle a century later.

As we move into the modern era, Schwartz describes how investigators demonstrated that these strange encephalopathies were transmissible, first in sheep, and then across the species barrier from sheep into goats (which revealed the existence of distinct scrapie prion strains) and from goats into mice. The infection of mice was a significant step forward, dramatically accelerating the pace of laboratory research. About this time, it also became clear that the infectious agent, whatever it was, was resistant to formaldehyde treatment, a property not shared by any known virus. This was an important clue, hinting that the nature of the infectious agent was unique. As the story unfolds, Schwartz discusses most of the major advances in prion biology until the recent past, including the formulation of the protein-only hypothesis. However, the author's analyses of what are often fairly complex scientific issues can at times be too superficial, brushing any loose ends under the carpet.

The public health and economic fallout from the BSE epidemic, and the accumulating evidence that variant Creutzfeldt-Jakob disease resulted from exposure to BSE prions, has been huge, particularly in Europe. It is perhaps a little disappointing that Schwartz does not dedicate more of the book to the many lessons that can be learned about the political (mis)handling of the crisis, particularly in the UK.

Although prions may not make the front pages of our newspapers as frequently as they have in the past, "The Disease," as Schwartz refers to it, has a habit of reappearing unexpectedly, and often with insidious consequences. The recent furor in Canada, where a single BSE-infected animal was identified, shows that prion diseases remain a significant menace against which we must be constantly vigilant. What the book aptly illustrates is that the gathering of scientific knowledge is probably our best hope for containing the prion diseases and for tackling the future problems that will assuredly come our way. 